(C) 2017, Elsevier. Licensed under the Creative Commons Attribution-NonCommercial-NoDerivatives 4.0 International http://creativecommons.org/licenses/by-nc-nd/4.0/

\title{
Reading on the right when there's nothing left? Probabilistic tractography reveals hemispheric asymmetry in pure alexia
}

\section{Michele Veldsman ${ }^{1,2}$}

Tobias Loetscher ${ }^{3}$

Amanda Wood ${ }^{4,5,6}$

Amy Brodtmann 2,6

${ }^{1}$ Nuffield Department of Clinical Neuroscience, University of Oxford, Oxford, UK

${ }^{2}$ Behavioural Neuroscience and Stroke Divisions, Florey Institute for Neuroscience and Mental Health, Melbourne, Australia

${ }^{3}$ University of South Australia, Adelaide, Australia

${ }^{4}$ School of Life and Health Sciences \& Aston Brain Centre, Aston University

${ }^{5}$ Clinical Sciences, Murdoch Childrens Research Institute, Parkville, Melbourne, Australia

${ }^{6}$ University of Melbourne, Parkville, Melbourne, Australia

Corresponding author: Michele Veldsman

Nuffield Department of Clinical Neuroscience, University of Oxford, John Radcliffe Hospital, Level 6, West Wing, OX3 9DU

Ph: +44 1865234632 ; Email: michele.veldsman@nden.ox.ac.uk

\section{Tobias Loetscher}

School of Psychology, University of South Australia GPO Box 2471 Adelaide Australia 5001 Phone: +61 (8) 8302 4098; Email: tobias.loetscher@unisa.edu.au

\section{Amanda Wood}


School of Life and Health Sciences \& Aston Brain Centre, Aston University, Aston Triangle, West Midlands, UK, B4 2ET

Phone: +44 (0) 1212043593 email: a.wood4@aston.ac.uk

\section{Amy Brodtmann}

The Florey Institute for Neuroscience and Mental Health, 245 Burgundy Street, Heidelberg, 3084 Australia; +6190357000; amy.brodtmann@,florey.edu.au

\section{Word count: 6179}

Key words: reading; alexia; diffusion tractography; stroke

Running head: Hemispheric asymmetry in pure alexia

\section{Acknowledgments}

Thanks to Shawna Farqurson for invaluable assistance with the scans, and to our participants for contributing so much of their time.

\section{Funding}

This work was supported by the National Health and Medical Research Council under Grant APP1020526. 


\begin{abstract}
We present a patient with reading inexpertise and right hemianopia following left posterior cerebral artery (PCA) stroke. We examine the extent of disruption to reading performance and the extent of white matter tract damage relative to a patient with more limited PCA infarction and isolated right hemianopia. We show white matter disconnection of the temporal occipital fusiform cortex in our pure alexia patient. Connectivity-based laterality indices revealed right hemisphere laterality in the alexia patient; this was not associated with improved reading function. We speculate that the degree of premorbid laterality may be a critical factor affecting the extent of reading dysfunction in alexia.
\end{abstract}




\section{Background}

Reading difficulties are common following temporo-occipital infarction arising from posterior cerebral artery (PCA) occlusion or embolism. In the majority of patients, reading difficulties are secondary to visual field deficits (Pflugshaupt et al., 2009). Rarely, patients exhibit the clinical syndrome of alexia without agraphia, or pure alexia, retaining the ability to write, spontaneously or to dictation, but unable to read what they've written. They exhibit profound lexical difficulties, often with "letter-by-letter" reading. Alexia without agraphia is regarded as one of the classical disconnection syndromes.

Cortical regions associated with pure alexia have remained under intense debate since its first description by Dejerine (1892). Most researchers agree that key components of the reading network lateralize to the left hemisphere (LH), around the temporo-occipital cortex (Dehaene \& Cohen, 2011). Localization of the cortical regions important for reading have been characterized with increasing precision using intracranial recording (Nobre, Allison, \& McCarthy, 1994), lesion mapping (Sebastian et al., 2014) and functional magnetic resonance imaging (fMRI, Dehaene \& Cohen, 2011). This has led to the proposition of the 'visual word form area (VWFA), a region with orthographic specialization essential for fluent and rapid reading (Dehaene \& Cohen, 2011). However, pure alexia can occur in the absence of complete VWFA damage when the region becomes disconnected as a result of damage to the inferior longitudinal fasciculus (ILF, Epelbaum et al., 2008). Whilst there is ongoing debate on the existence (Dehaene \& Cohen, 2011) versus the 'myth' (Price \& Devlin, 2011), of the VWFA, there is agreement that the study of patients with alexia requires detailed understanding of the white matter connections within the temporo-occipital region, specialized and likely lateralized to the LH (Behrmann \& Plaut, 2015; Bouhali et al., 2014; Yeatman, Rauschecker, \& Wandell, 2013). 
Lateralization and specialization of reading in the brain may arise as a result of the proximity to retinotopic cortices and left-lateralized language functions that increases pathway and network efficiency (Behrmann \& Plaut, 2015; Yeatman et al., 2013). Behrmann and Plaut (2015) propose a graded, rather than binary, lateralization of reading function, arising dynamically during childhood with the acquisition of language and reading ability. This model emphasizes the importance of the extent of laterality, and acknowledges some right hemisphere $(\mathrm{RH})$ involvement in the reading network. A bilateral reading network, even if asymmetrically biased to the $\mathrm{LH}$, would be more robust to damage as a result of stroke or brain injury and offers greater promise for recovery. This might predict that greater RH involvement would be associated with better recovery of reading ability, yet there has been little evidence of recovery from alexia based on RH compensatory activity (Behrmann \& Plaut, 2015).

In other stroke syndromes, recovery is associated with restitution of peri-lesional cortex, rather than compensatory activity in homologous contralesional regions (Calautti et al., 2010; Grefkes \& Fink, 2011). For example, in patients with unilateral damage to motor cortex, a shift to contralesional hemispheric utilization is associated with poorer outcome, especially if sustained (Calautti et al., 2010). This has led to the use of laterality indices - typically measured with fMRI - to provide a metric for the degree of intra-hemispheric shift in function in recovery from stroke. Laterality indices are particularly useful in relation to unilaterally dominant functions, such as language, where bias to the preferred hemisphere is established and indicative of healthy function (Thulborn, Carpenter, \& Just, 1999). 
Two other sources of evidence suggest RH compensation may be maladaptive in alexia, as in other stroke syndromes. Firstly, inducing LH lateralization in healthy adult, below average readers using transcranial direct current stimulation (tDCS) improves reading efficiency by reducing excitability of the RH (Turkeltaub et al., 2012). Secondly, audio-visual training improves reading performance and functional connectivity in perilesional LH in pure alexia patients and reduces connectivity in the RH (Woodhead et al., 2013). This suggests LH laterality is critical for proficient reading.

We examined the extent of white matter tract damage and diffusion-derived laterality indices (LI) as a measure of hemispheric lateralization in two stroke patients and nine healthy controls. Patient A suffered a large PCA infarct affecting occipital and hippocampal cortex, involving some of the splenium. In Patient B, infarction was limited to the occipital region. We studied these two patients with behavioral tests of reading ability, fMRI and diffusion tractography. We hypothesized that Patient A would demonstrate a rightward laterality, corresponding to her profound reading deficits as she attempted to "read on the right" (Coslett \& Monsul, 1994).

\section{Methods}

\section{Participants}

Single case data were taken from a prospective clinical stroke recovery study. The healthy control for the behavioral and fMRI component was recruited from a previously identified pool of volunteers. Patients were recruited from the Austin Health Stroke Unit, with ethical approval obtained from the hospital's ethical review board.

\section{Case report patient $A$}


A 63 year-old right handed primary school teacher with 20 years teaching experience and an undergraduate and postgraduate degree, presented with a word finding difficulty, slurring of speech, and visual disturbance on a background of stroke risk factors. She was noted to have a nominal aphasia, right homonymous hemianopia, and right visual neglect. She had an extensive past medical history, including Type 2 diabetes mellitus, hypertension, congestive cardiac failure, obstructive sleep apnoea, and depression. Initial CT brain scan confirmed an acute PCA infarct with hemorrhagic transformation. Follow up CT imaging after 14 days of language difficulties confirmed extension of her PCA stroke (Fig 3). She received 6 weeks of intensive inpatient rehabilitation. On discharge, she was still noting mild word finding difficulty but profound reading problems, including an inability to read any text. At time of testing, this had improved to the point of being able to read slowly "letter by letter". She said she was able to write but unable to read what she had written. When seen for participation in this study nine months after her acute event, she still had a significant hemianopia. She stated that there had been a functional improvement in reading with scanning strategies learnt in rehabilitation, but she was unable to return to prior work or driving. She was found to have pure alexia with letter-by-letter reading.

At time of testing, Patient A had intact comprehension and fluent speech with only intermittent word finding problems. She scored 69 out of 100 points in a brief cognitive test battery (ACE-R, Mioshi et al., 2006) with difficulties in memory, fluency, confrontation naming and reading. In contrast to her reading abilities, writing was relatively preserved. She wrote a correct sentence in the ACE-R, on dictation of single words she had 5 errors out of 23 words (Referree, redicule, visiter, revench (revenge), cayotric (chaotic)). The patient's visuospatial and perceptual abilities were largely intact. Medmont M600 (Camberwell, 
Australia) automated perimetry full visual field testing (including fixation loss errors) revealed an homonymous hemianopia.

\section{Case report patient $B$}

A 58 year-old right handed woman presented after headache and visual symptoms failed to resolve after 2 days, with a medical history of autoimmune hepatitis, migraine, smoking (40 pack years), prior transient ischemic attack (left sensory change), hypercholesterolemia and hypertension. She had a strong family history of vascular disease. On admission, a right homonymous hemianopia was diagnosed. CT brain scan revealed a subacute left occipital stroke. Transcranial Doppler revealed a small calibre left vertebral artery with severe proximal stenosis. She was discharged on day 3 for outpatient rehabilitation. A functional vision assessment by Guide Dogs Australia found she commenced testing "on the right of center and worked to the far right side before working systematically across to the left perimeter. She maintained this pattern as tasks became more complex". After extensive outpatient rehabilitation, a Goldmann visual field assessment revealed significant improvement in her hemianopia to the point she was able to return to driving 5 months after her stroke.

Patient B had 18 years of education, having completed a 5-year combined undergraduate degree and Masters program. At time of testing, she scored 93/100 points in the ACE-R with some difficulties in the memory recall, but no obvious impairments in language, reading and writing. Perimetry with static stimuli revealed a residual incomplete right homonymous hemianopia (mainly right inferior quadrantanopia).

\section{Healthy control participant}


A 61 year-old right handed, education-level matched woman with no significant medical history or stroke risk factors was included for comparison and underwent identical testing. She scored 98/100 points in the ACE-R. A further 9 healthy, right-handed control participants (4 female, mean age 69 years, SD 7.04) with no significant medical history or stroke risk factors were included in the diffusion component for comparison with the patients.

\section{Procedure}

\section{Reading task}

The reading task was presented on a Dell 14-inch screen laptop running E-prime software (Psychology Software Tools, Inc.). Twenty-four 3, 5 or 7 letter words, matched for frequency and imageability, were drawn from the MRC psycholinguistic database (Coltheart, 2007). Words were presented in upper case letters (50-point Arial script), one at a time centred on the left half of the screen. Participants were instructed to read words aloud as quickly and accurately as possible, with a maximum response time of 25 seconds per word. Naming time and accuracy were recorded for subsequent analysis.

\section{Word Comparison}

216 word pairs between 5-7 letters in length, matched in imageability and frequency, were derived from (Mycroft, Behrmann, \& Kay, 2009) word pair list. Each word pair comprised either identical items (108 word pairs) or items differing by a single letter at the beginning, middle, or end of the word (36 word pairs each, totalling in 108 pairs). Pairs were presented in capital letters (50-point) centred on the left half of the screen. Participants were instructed to indicate as quickly as possible whether the word pairs were identical or different. Time from word presentation to response was recorded.

\section{Functional and Structural Imaging}


Three letter words from the reading task, three letter strings, checkerboards and crosshairs were presented in a block design on a computer screen that was projected onto a mirror attached to the MRI scanner head coil. Images were displayed for 4 seconds, to allow sufficient time for letter-by-letter reading, in 24 second blocks in a single 6.6 minute run (Fig $1)$.

$<$ Fig 1 here $>$

Fig 1 Schematic of block design of fMRI task

Brain images were collected on a Siemens 3T Tim Trio scanner (Erlangen, Germany) with a 32-channel head coil at the Brain Research Institute, Melbourne, Australia. A 192-slice a Magnetization-Prepared Rapid Acquisition (MPRAGE) with $0.9 \mathrm{~mm}$ isotropic voxels, $230 \mathrm{~mm}$ FOV, 256x256 matrix, $1.9 \mathrm{sec}$ TR, 0.9sec TI, 2.6ms TE was acquired. A 3D SPACE- Fluid Attenuated Inversion Recovery (FLAIR) image was acquired with $1 \mathrm{~mm}$ isotropic voxels, 256mm FOV, 256x256 matrix, $6 \mathrm{sec}$ TR, 2.1sec TI, 389ms TE. 132 volumes of 44interleaved slice echo-planar images were acquired with $3 \mathrm{~mm}$ isotropic voxels, $504 \mathrm{~mm}$ FOV, $72 \times 72$ matrix, $3 \mathrm{sec}$ TR, 30ms TE, $85^{\circ}$ flip angle. 60 volumes of single-shot, spin-echo, echo planar images were collected at a TR of 8.4 seconds, TE $110 \mathrm{~ms}$, with 60 diffusion sensitization directions, $b=3000 \mathrm{~s} / \mathrm{mm}^{2}$ and a $2.5 \mathrm{~mm}$ isotropic voxel size $(240 \mathrm{~mm}$ FOV, 96x96 matrix). For the healthy control participants, additional $b=0$, reverse-phase encoded images were acquired, with otherwise identical parameters, to aid correction for geometric distortion.

\section{Functional Image preprocessing and analysis}


Functional image preprocessing included slice-timing correction, realignment and coregistration to the structural MPRAGE using SPM8 (http://www.fil.ion.ucl.ac.uk/spm/). Patient lesions were manually traced to create lesion masks, which were inspected and verified by stroke neurologist $\mathrm{AB}$. Lesion masks guided segmentation and normalization of patient structural images to MNI template space using the Clinical Toolbox (Rorden, Bonilha, Fridriksson, Bender, \& Karnath, 2012). Functional images were smoothed with a $8 \mathrm{~mm}$ FWHM Gaussian kernel. A general linear model of boxcar functions was fitted to all voxels. Six nuisance regressors were included in the model to account for head movement. A $128 \mathrm{~s}$ cut-off high pass filter was applied to the data to remove low frequency noise.

\section{Diffusion image preprocessing and analysis}

Diffusion images were preprocessed using the Diffusion Toolbox (FDT) of FSL (Behrens, Berg, Jobadi, Rushworth, \& Woolrich, 2007). FSL's 'topup' function was used to estimate susceptibility induced distortions and inform the 'eddy' function, which corrects for gradient coil distortions and movement.

Binarised seed masks were created from the probabilistic, lateralized Harvard-Oxford Cortical Structural Atlas built into FSL. Seed regions were determined by significant activation identified in the reading and letter-string fMRI task. A region in the middle frontal gyrus served as a control region. Given there is no damage to this region in either patient, tractography should be similar to the healthy controls. Left and right hemisphere masks were created by thresholding voxels with greater than 75 percent probability of belonging to the region of interest on a probabilistic map. 
FDT's Bayesian Estimation of Diffusion Parameters Obtained using Sampling Techniques for modelling Crossing Fibres (Bedpostx) estimated the probability distribution of fibre orientations on a voxelwise basis (Behrens et al., 2007). For each voxel per seed, 5000 streamlines were sent with a default curvature threshold of 0.2 and a step length of $0.5 \mathrm{~mm}$. The binary lesion mask was used as an exclusion mask in the patients. Probabilistic tractography maps were thresholded to include only 0.0025 percent of voxels with the highest probability of connectivity to the seed mask. This conservative estimate removes voxels with a low probability of connectivity to the seed mask. Group maps were created from the healthy control subjects by adding the thresholded tract maps for each healthy participant and then thresholding the resulting group map to include only voxels in which at least 30 percent of the group contained the given tract.

Given normal variability in white matter tracts across subjects, even in the absence of lesion damage, and the inherent limitations of estimating white matter disruption on a single patient basis, we compared tracts across hemispheres using a laterality index. The laterality index was calculated as Volume $\left.\left({ }_{\mathrm{LH}}\right)-\operatorname{Volume}\left({ }_{\mathrm{RH}}\right) /\left(\operatorname{Volume}_{(\mathrm{LH}}\right)+\operatorname{Volume}\left({ }_{\mathrm{RH}}\right)\right)$; where $\mathrm{LH}$ and $\mathrm{RH}$ denote left and right hemisphere.

To further quantify hemispheric differences, we compared the volume of the tract derived from the TOFC seed in each hemisphere in the patients with the healthy controls. We used single case methodology, which treats the controls as statistics rather than population parameters (Crawford \& Howell, 1998). The test provides a point estimate of the abnormality of a score, that is the percentage of the population with scores (or in this case tract volume) below the patient, and $95 \%$ confidence limits. 


\section{Results}

\section{Reading Task}

Reading performance for the healthy control participant and Patient B was errorless. Patient A read all 3-letter words accurately, but struggled to read the 5 and 7-letter words (error rates of $21 \%$ and $46 \%$, respectively). Reading difficulties were also reflected in reading time. Patient A was roughly 10 times slower in reading the words than the control and Patient B (Patient A: mean reading time 13.38s, standard deviation $(\mathrm{SD})=6.31$; Patient $\mathrm{B}$ : mean=1.37s, $\mathrm{SD}=2.78$; Healthy Control: mean=1.04s, $\mathrm{SD}=3.11$ ). Reading time was modulated by word length (Fig 2a). As determined by linear regression, Patient A's reading time increased by $2.5 \mathrm{~s}$ per additional letter. This increase is substantially larger than the additional time requirements of the controls $(0.63 \mathrm{~s}$ and $0.23 \mathrm{~s}$ reading time increase per additional letter for Patient B and the Healthy Control, respectively).

\section{Word Comparison}

Patient A felt exhausted towards the end of the task and completed 200 of the 216 trials. Error rates were $2.5 \%, 1.8 \%$ and $1.3 \%$ for Patient A, Patient B, and the healthy control, respectively. Patient A was considerably slower (mean correct responses $=4.69 \mathrm{~s}, \mathrm{SD}=2.15$ ) than Patient B (mean RT=1.80s, $\mathrm{SD}=6.38$ ) and the healthy control (mean $\mathrm{RT}=1.09 \mathrm{~s}$, $\mathrm{SD}=3.56$ ). In Patient A, the response time was noticeably modulated by the position of difference between the two stimuli words (Fig 2b).

$<$ Fig 2 here $>$

Fig 2 Behavioral performance (reaction time) in the a) reading task and b) the word comparison task. Error bars indicate standard error 


\section{Structural MRI findings}

Structural images revealed chronic infarction affecting left occipital and mesial temporal cortex; lingual and fusiform gyri and most of the left hippocampus in Patient A (Fig 3). Patient A's FLAIR image also revealed splenial changes. A left chronic occipital infarct was apparent on T1 imaging of Patient B, with no hippocampal or splenial infarction (Fig 4).

$<$ Fig 3 here $>$

Fig 3 Patient A, extent of lesion shown in red on T1 image

$<$ Fig 4 here $>$

Fig 4 Patient B, extent of lesion shown in red on T1 image

\section{Functional imaging}

$<$ Fig 5 here $>$

Fig 5 Statistical parametric maps of word (left) and letter-string (middle) and checkerboard (right) activation in the fMRI task in the healthy control volunteer. Color map indicated tvalue at height threshold $p<0.01$, in contrasts against implicit baseline

In the healthy control participant, activity associated with word and letter reading was bilateral in the lateral occipital cortex and fusiform gyrus, extending into the lingual gyrus and encompassing the occipital poles in contrasts against implicit baseline (Fig 5). There was no detectable difference in activation associated with words compared to letter-strings in the healthy control at height threshold $\mathrm{p}<0.01$ (uncorrected). In the checkerboard blocks, when 
contrasted against implicit baseline, activity was spatially similar to the word and letter string blocks but included more posterior regions including low-level visual areas of the occipital poles and calcarine sulcus. Direct contrasts of word and letter string activation against checkerboard showed no significant detectable activation at (height threshold, $\mathrm{p}<0.01$, uncorrected). Patient A experienced nausea and kept her eyes closed for most of the fMRI scan, therefore the key comparison of reading activity between Patient A, Patient B and the healthy control could not be reliably estimated.

We used activity in the word and letter-string blocks in the healthy control participant to guide our choice of regions for the tractography and laterality analysis. Seed regions of interest included the temporal occipital fusiform gyrus (TOFC, encompassing the visual word form area), the lateral occipital cortex (LOC), the lingual gyrus (LG), the occipital pole (OP) and the middle frontal gyrus (MFG).

\section{Diffusion Tractography}

Probabilistic tractography initiated from the TOFC seeds in the right and left hemisphere showed severe disruption to white matter connectivity in Patient A and relatively preserved tracts in Patient B compared to the 9 healthy control participants (Fig 6). The estimated tracts included the inferior longitudinal fasciculus (ILF) and the inferior fronto-occipital fasciculus (IFOF, Catani \& Thiebaut de Schotten, 2008).

$<$ Fig 6 here $>$ 
Fig 6 Probabilistic tractography seeded from the temporal occipital fusiform cortex in a.) Patient A, b.) Patient B and c.) the healthy control group. Left hemisphere tracts shown in blue, right hemisphere in orange

Middle frontal gyrus seeds produced tracts in Patient A that were very similar to the healthy controls, providing some evidence of specificity in the tract disruption to the TOFC (Fig 7).

$<$ Fig 7 here $>$

Fig 7 Probabilistic tractography seeded from the middle frontal gyrus in a.) Patient A, b.) Patient $B$ and c.) the healthy control group. Left hemisphere tracts shown in blue, right hemisphere in orange

Using tract volume we calculated a laterality index of key regions of the reading network. There were normal levels of laterality within the lateral occipital cortex, lingual gyrus, occipital poles and middle frontal gyrus (+/- 0.2) in Patient B and the healthy controls (Fig 8). Notably, there is strong RH laterality in the tracts derived from the TOFC seed in Patient A. Using single case methodology, for the LH seeded tract, 3.62\% [95\% confidence limits: 0.03 $17.66 \%$ ] of the population fell below Patient A's tract volume compared to $90.78 \%$ [CI: 70.07\%-99.37\%] in Patient B. This provides a quantitative estimate of the disruption to the ILF and the IFOF tracts (Fig 6) and further quantifies the leftward laterality observed in Patient B in line with the healthy controls. For the RH TOFC seeded tract, $41.82 \%$ [CI: $18.93-67.15 \%]$ of the population fell below the tract volume of Patient A. This was almost double the abnormality point estimate in Patient B which indicated $82.82 \%$ [CI: $58.38 \%$ - 
96.93\%] of the population had tract volumes below that observed for their RH ILF and the IFOF.

$<$ Fig 8 here $>$

Fig 8 Laterality index based on volume of tracts for regions of interest in Patient A, Patient B and 9 healthy control volunteers. Error bars indicated standard error. Negative values indicate greater volume in the right hemisphere. Temporal occipital fusiform cortex (TOFC); Lateral occipital cortex (LOC); Lingual gyrus (LG); Occipital pole (OP); Middle frontal gyrus $(M F G)$

\section{Discussion}

We present behavioral and neuroimaging characterization of a patient with pure alexia and discuss the implications for hemispheric specialization of language and recovery of reading function. Patient A showed clear evidence of pure alexia as demonstrated by damage to the left temporo-occipital region encompassing the VWFA; a word-length effect in reading, relatively intact writing and other language functions; and damage to the ILF and the IFOF. These are key factors distinguishing pure alexia from other reading difficulties (Starrfelt \& Shallice, 2014). The word-length effect in Patient A was pronounced, increasing by $2.5 \mathrm{~s}$ per additional letter, suggesting a severe form of letter-by-letter reading. It is noteworthy that testing took place 9 months after the patient suffered the infarct and that the patient was initially unable to read any words.

In our fMRI task, activity associated with reading was evident in temporo-occipital regions including the fusiform and lingual gyrus in the healthy control participant. This activity was 
bilateral (Ossowski \& Behrmann, 2015) and in regions associated with the reading network (Vigneau, Jobard, Mazoyer, \& Tzourio-Mazoyer, 2005). The activity also replicates that seen in an fMRI reading task of an alexia patient and healthy controls (Welcome, Pasquarella, Chen, Olson, \& Joanisse, 2014), in which fMRI activity was relatively preserved in the alexia patient, including within the VWFA, but diffusion tensor imaging revealed disconnection of the ventral temporal cortex. Due to intolerance to the fMRI scanning, we were unable to reliably estimate functional activity in our alexia patient. However, in line with this previous work we also observed disconnection in the temporo-occipital region (Epelbaum et al., 2008; Welcome et al., 2014). Using a seed in the temporal-occipital fusiform (TOFC) region, that encompassed the VWFA, we showed white matter connections overlapping with major tracts of the ILF and the IFOF. These are key tracts known to connect the occipital lobe to the medial temporal lobe and the inferior frontal cortex regions of the reading network (Yeatman et al., 2013). The volume of the tracts was noticeably reduced in our alexia patient compared to the patient with isolated hemianopia and the healthy controls, and this was most clear in the LH. Probabilistic tractography seeded from the healthy middle frontal gyrus in both patients showed preserved tracts that were qualitatively very similar to the healthy controls, providing evidence of the specificity of tract disruption as a result of disconnection due to stroke infarction in our pure alexia patient.

Using a laterality index, we found marked rightward laterality in our alexia patient in tracts seeded from the TOFC. In Patient B, left laterality was evident in the TOFC and similar to the healthy control participants, in line with Patient B's relatively preserved reading performance compared to Patient A - who showed clear evidence of letter-by-letter reading. Laterality in the other regions of interest was within the normal range of variation expected (Seghier, 2008). 
Tracts estimated from the TOFC in the right hemisphere of Patient A were notably smaller than Patient B, and the healthy controls, despite this region being unaffected by stroke infarction. By contrast, tracts estimated in Patient B showed little asymmetry between hemispheres, as was also evident in the healthy controls, and quantified by the laterality indices. An abnormality point estimate further quantified the lateralised tract volume in Patient A and B compared to healthy controls. This demonstrated severely disrupted LH tract volume (Fig 6) which is expected given damage in the TOFC in Patient A. In the unaffected $\mathrm{RH}$, the abnormality point estimated indicated Patient A was in the bottom $42 \%$ of ILF/IFOF tract volumes of the normal population. Although this is within a normal range, it is notable that the abnormality point estimate in Patient B indicated a RH tract volume greater than $83 \%$ of the normal population. It may be a premorbid strongly left lateralized reading network that resulted in Patient A showing little evidence of recovery from her reading deficits 9 months after stroke. This asymmetry is unlikely to be the result of the tract estimation as the same analysis seeded from the middle frontal gyrus region showed no evidence of asymmetry. Welcome et al., (2014) present a case of alexia in a patient who showed similar asymmetry in tracts in the right hemisphere. Their patient was a journalist and novelist and they speculate that the patient's extensive experience with reading and writing may have resulted in a strongly left lateralized reading network and under-developed white matter connectivity in the RH. Similarly, prior to her stroke, Patient A was an avid reader, teaching literature as part of her work.

There is some evidence of a shift to RH laterality in recovery from aphasia (Thulborn et al., 1999). The RH may have a compensatory role, as demonstrated by the recruitment of regions within the nondominant hemisphere in aphasia recovery (Thiel et al., 2001). In line with this, 
transcranial magnetic stimulation to disrupt unilateral function affected oral reading ability in an alexia patient when it was applied to the right, but not the left hemisphere (Coslett \& Monsul, 1994). Similarly, a patient with a left occipito-temporal hematoma lost all residual reading ability after a subsequent mirror-image, RH hematoma (Bartolomeo, Bachoud-Lévi, Degos, \& Boller, 1998). In both cases, this suggests the RH had a beneficial or compensatory role in reading ability. Damage to the LH may result in unmasking of latent $\mathrm{RH}$ reading functions or compensatory reorganization of the RH as a result of stroke (Hamilton, Chrysikou, \& Coslett, 2011, Forkel et al., 2014) . On the other hand, there may be a dysfunctional shift in language processing to the contralesional hemisphere (Hamilton et al., 2011). The evidence may be mixed because the role of the RH changes in the course of recovery (Gainotti, 2015). In the acute stages of post-stroke aphasia, language improvement correlates with activity in the $\mathrm{RH}$, but with restitution of activity in perilesional LH in the chronic stage (Saur et al., 2006). Hamilton et al., (2011) acknowledge that the role of the RH is likely to change across the course of recovery and its role likely depends on the extent of LH damage. In other stroke syndromes, RH compensatory activity appears to be maladaptive (Grefkes \& Fink, 2011).

We reasoned that the extent of right lateralization may be important in characterizing residual reading ability in pure alexia. The degree of left lateralization of reading function is associated with increasing literacy (Dehaene et al., 2010; Dehaene, Cohen, Morais, \& Kolinsky, 2015), acquisition of language skills, word recognition and reading ability (Behrmann \& Plaut, 2015). This suggests that left hemispheric specialization is a key feature of reading ability. Inducing LH lateralization using tDCS improves reading efficiency, likely through reducing excitability of the RH (Turkeltaub et al., 2012) and reading training results in increased functional connectivity of the reading network in the $\mathrm{LH}$ in alexia patients and 
decreased connectivity in the RH (Woodhead et al., 2013). There is evidence of lateralization of function in other cognitive domains, including visuospatial and phonological processing and in all vertebrate species (Hervé, Zago, Petit, Mazoyer, \& Tzourio-Mazoyer, 2013). This suggests hemispheric lateralization, or specialization, is an efficient form of functional organization (Hervé et al., 2013). In their graded hemispheric lateralization hypothesis, Behrmann and Plaut (2015) suggest there may be differences in cooperation and competition across hemispheres that results in varying degrees of lateralization across individuals. Their theory makes the prediction that individuals who have strongly lateralized language function, will have minimal representation of language in the right hemisphere and may therefore have little hope for recovery of function when the preferred hemisphere is damaged (Behrmann \& Plaut, 2015). Lateralization of reading function likely arises over the course of development as the result of a general set of principles that constrain hemispheric organization (Behrmann \& Plaut, 2015). Individual differences in neuroanatomy may also constrain the degree of lateralization of reading function. Anatomical hemispheric asymmetries emerge early in development, likely as the result of genetic factors (Hervé et al., 2013). Left lateralization may be associated with reading proficiency because having orthographic representations in close proximity to language areas results in short interhemispheric connections; a more efficient network organisation (Bullmore \& Sporns, 2009). Strong lateralization is likely to be relatively rare, which also accounts for the rare cases of persistent alexia after stroke. This theory fits our current data, in which despite showing clear rightward lateralization in key tracts of the reading network, our pure alexia patient demonstrated little evidence of recovery of reading function 9 months after stroke.

\section{Limitations}


Limited inferences can be drawn from single subject data, a common problem in case studies with relatively rare stroke syndromes. Premorbid data in stroke studies, especially in rare stroke syndromes such as alexia are exceedingly rare. We did not have premorbid data so we were not able to directly estimate the degree of hemispheric laterality pre and post-stroke. We attempted to quantify lateralisation by comparing tract volume to the normal control population with the assumption that low volume compared to the normal population would indicate a degree of lateralisation to the opposite hemisphere. However, lateralisation is best compared as the difference between hemispheres within subjects. We used a lateralisation index to quantify the degree of lateralisation within subjects. Nevertheless, without premorbid data we are only able to speculate on the degree of hemispheric specialisation and its role in recovery.

Due to the intolerance to the task in the fMRI scanner, we were unable to examine brain activity in response to reading in our alexia patient and therefore we cannot equivocally state whether reading impairments in Patient A are the result of the stroke infarction itself or disconnection as a result of damaged white matter tracts.

Probabilistic tractography is limited in its ability to estimate white matter tracts to the extent that allows for specific anatomical hypotheses regarding cortical disconnection (Soares, Marques, Alves, \& Sousa, 2013). Our diffusion sequences were optimized acquisitions with a high $b$-value (3000 s/mm ${ }^{2}$ ), 60 encoding directions and high in-plane resolution in order to maximize our signal to noise ratio and spatial resolution (Mukherjee, Berman, Chung, Hess, \& Henry, 2008; Soares et al., 2013). The technique does not allow inferences to be drawn on specific tracts, therefore we have limited our inferences to the residual volume of major tracts and used laterality indices as a proxy for white matter changes relative to healthy controls. 
Laterality indices in fMRI studies can be subject to several sources of bias, including the choice of task and the baseline against which activity is measured (Seghier, 2008). In contrast structural measures can provide a more easily interpretable measure of hemispheric lateralization. Nevertheless there is good correspondence between laterality as measured with fMRI and laterality based on tractography measures in language pathways (Powell et al., 2006).

\section{Conclusions}

Diffusion tractography derived laterality indices revealed rightward laterality of key reading network tracts in our pure alexia patient, associated both with a larger infarct and poor reading recovery. A patient with isolated hemianopia, but preserved reading ability, showed preserved left lateralization similar to stroke-free age-matched control participants with normal reading ability. Premorbid lateralization of function may be a key indicator of likelihood of recovery of function. We provide support for the importance of left lateralization of white-matter connectivity underlying reading proficiency.

\section{Informed consent:}

All procedures followed were in accordance with the ethical standards of the responsible committee on human experimentation (institutional and national) and with the Helsinki Declaration of 1975, and the applicable revisions at the time of the investigation. Informed consent was obtained from all patients for being included in the study. Additional informed consent was obtained from all patients for whom identifying information is included in this article. 


\section{Conflict of interest}

Michele Veldsman, Amy Brodtmann, Amanda Wood and Tobias Loetscher declare that they

have no conflict of interest.

\section{References}

Bartolomeo, P., Bachoud-Lévi, A. C., Degos, J. D., \& Boller, F. (1998). Disruption of residual reading capacity in a pure alexic patient after a mirror-image right-hemispheric lesion. Neurology, 50(1), 286-8.

Behrens, T. E. J., Berg, H. J., Jbabdi, S., Rushworth, M. F. S., \& Woolrich, M. W. (2007). Probabilistic diffusion tractography with multiple fibre orientations: What can we gain? NeuroImage, 34(1), 144-55. https://doi.org/10.1016/j.neuroimage.2006.09.018

Behrmann, M., \& Plaut, D. C. (2015). A vision of graded hemispheric specialization. Annals of the New York Academy of Sciences. https://doi.org/10.1111/nyas.12833

Bouhali, F., Thiebaut de Schotten, M., Pinel, P., Poupon, C., Mangin, J.-F., Dehaene, S., \& Cohen, L. (2014). Anatomical Connections of the Visual Word Form Area. Journal of Neuroscience, 34(46), 15402-15414. https://doi.org/10.1523/JNEUROSCI.491813.2014

Bullmore, E., \& Sporns, O. (2009). Complex brain networks: graph theoretical analysis of structural and functional systems. Nature Reviews. Neuroscience, 10(3), 186-98. https://doi.org/10.1038/nrn2575

Calautti, C., Jones, P. S., Naccarato, M., Sharma, N., Day, D. J., Bullmore, E. T., ... Baron, J.-C. (2010). The relationship between motor deficit and primary motor cortex hemispheric activation balance after stroke: longitudinal fMRI study. Journal of Neurology, Neurosurgery, and Psychiatry, 81(7), 788-92. https://doi.org/10.1136/jnnp.2009.190512

Catani, M., \& Thiebaut de Schotten, M. (2008). A diffusion tensor imaging tractography atlas for virtual in vivo dissections. Cortex, 44(8), 1105-1132. https://doi.org/10.1016/j.cortex.2008.05.004

Coltheart, M. (2007). The MRC psycholinguistic database. The Quarterly Journal of Experimental Psychology Section A, 33(4), 497-505. https://doi.org/10.1080/14640748108400805

Coslett, H. B., \& Monsul, N. (1994). Reading with the right hemisphere: evidence from transcranial magnetic stimulation. Brain and Language, 46(2), 198-211. https://doi.org/10.1006/brln.1994.1012

Crawford, J. R., \& Howell, D. C. (1998). Comparing an Individual's Test Score Against Norms Derived from Small Samples. The Clinical Neuropsychologist (Neuropsychology, Development and Cognition: Section D), 12(4), 482-486. https://doi.org/10.1076/clin.12.4.482.7241

Dehaene, S., \& Cohen, L. (2011). The unique role of the visual word form area in reading. Trends in Cognitive Sciences, 15(6), 254-62. https://doi.org/10.1016/j.tics.2011.04.003

Dehaene, S., Cohen, L., Morais, J., \& Kolinsky, R. (2015). Illiterate to literate: behavioural and cerebral changes induced by reading acquisition. Nature Reviews Neuroscience, 16(4), 234-44. https://doi.org/10.1038/nrn3924

Dehaene, S., Pegado, F., Braga, L. W., Ventura, P., Nunes Filho, G., Jobert, A., ... Cohen, L. 
(2010). How learning to read changes the cortical networks for vision and language. Science (New York, N.Y.), 330(6009), 1359-1364.

https://doi.org/10.1126/science.1194140

Dejerine, J. (1892). Contribution a l'etude anatomoclinique et clinique des differentes varietes de cecite verbal. CR Hebdomadaire Des Sceances et Memories de La Societe de Biologie, 4, 61-90.

Epelbaum, S., Pinel, P., Gaillard, R., Delmaire, C., Perrin, M., Dupont, S., ... Cohen, L. (2008). Pure alexia as a disconnection syndrome: new diffusion imaging evidence for an old concept. Cortex; a Journal Devoted to the Study of the Nervous System and Behavior, 44(8), 962-74. https://doi.org/10.1016/j.cortex.2008.05.003

Gainotti, G. (2015). Contrasting opinions on the role of the right hemisphere in the recovery of language. A critical survey. Aphasiology, 7038(July 2015), 1-18. https://doi.org/10.1080/02687038.2015.1027170

Grefkes, C., \& Fink, G. R. (2011). Reorganization of cerebral networks after stroke: new insights from neuroimaging with connectivity approaches. Brain: A Journal of Neurology, 134(Pt 5), 1264-76. https://doi.org/10.1093/brain/awr033

Hamilton, R. H., Chrysikou, E. G., \& Coslett, B. (2011). Mechanisms of aphasia recovery after stroke and the role of noninvasive brain stimulation. Brain and Language, 118(1), 40-50. https://doi.org/10.1016/j.band1.2011.02.005

Hervé, P. Y., Zago, L., Petit, L., Mazoyer, B., \& Tzourio-Mazoyer, N. (2013). Revisiting human hemispheric specialization with neuroimaging. Trends in Cognitive Sciences, 17(2), 80. https://doi.org/10.1016/j.tics.2012.12.004

Mioshi, E., Dawson, K., Mitchell, J., Arnold, R., Hodges, J. R., Steunenberg, B., ... Kerkhof, A. J. F. M. (2006). The Addenbrooke's Cognitive Examination Revised (ACE-R): a brief cognitive test battery for dementia screening. International Journal of Geriatric Psychiatry, 21(12), 1078-1085. https://doi.org/10.1002/gps

Mukherjee, P., Berman, J. I., Chung, S. W., Hess, C. P., \& Henry, R. G. (2008). Diffusion tensor MR imaging and fiber tractography: Theoretic underpinnings. American Journal of Neuroradiology, 29(4), 632-641. https://doi.org/10.3174/ajnr.A1051

Mycroft, R. H., Behrmann, M., \& Kay, J. (2009). Visuoperceptual deficits in letter-by-letter reading? Neuropsychologia, 47(7), 1733-44. https://doi.org/10.1016/j.neuropsychologia.2009.02.014

Nobre, A. C., Allison, T., \& McCarthy, G. (1994). Word recognition in the human inferior temporal lobe. Nature, 372, 260-263. https://doi.org/10.1038/372260a0

Ossowski, A., \& Behrmann, M. (2015). Left hemisphere specialization for word reading potentially causes, rather than results from, a left lateralized bias for high spatial frequency visual information. Cortex; a Journal Devoted to the Study of the Nervous System and Behavior, 72, 27-39. https://doi.org/10.1016/j.cortex.2014.12.013

Pflugshaupt, T., Gutbrod, K., Wurtz, P., Von Wartburg, R., Nyffeler, T., de Haan, B., ... Mueri, R. M. R. M. (2009). About the role of visual field defects in pure alexia. Brain, 132(7), 1907-17. https://doi.org/10.1093/brain/awp141

Powell, H. W. R., Parker, G. J. M., Alexander, D. C., Symms, M. R., Boulby, P. A., WheelerKingshott, C. A. M., ... Duncan, J. S. (2006). Hemispheric asymmetries in languagerelated pathways: a combined functional MRI and tractography study. NeuroImage, 32(1), 388-99. https://doi.org/10.1016/j.neuroimage.2006.03.011

Price, C. J., \& Devlin, J. T. (2011). The Interactive Account of ventral occipitotemporal contributions to reading. Trends in Cognitive Sciences, 15(6), 246-253. https://doi.org/10.1016/j.tics.2011.04.001

Rorden, C., Bonilha, L., Fridriksson, J., Bender, B., \& Karnath, H.-O. (2012). Age-specific 
CT and MRI templates for spatial normalization. NeuroImage, 61(4), 957-65.

https://doi.org/10.1016/j.neuroimage.2012.03.020

Saur, D., Lange, R., Baumgaertner, A., Schraknepper, V., Willmes, K., Rijntjes, M., \&

Weiller, C. (2006). Dynamics of language reorganization after stroke. Brain, 129(6), 1371-1384. https://doi.org/10.1093/brain/awl090

Sebastian, R., Gomez, Y., Leigh, R., Davis, C., Newhart, M., \& Hillis, A. E. (2014). The roles of occipitotemporal cortex in reading, spelling, and naming. Cognitive Neuropsychology, 31(5-6), 511-28. https://doi.org/10.1080/02643294.2014.884060

Seghier, M. L. (2008). Laterality index in functional MRI: methodological issues. Magnetic Resonance Imaging, 26(5), 594-601. https://doi.org/10.1016/j.mri.2007.10.010

Soares, J. M., Marques, P., Alves, V., \& Sousa, N. (2013). A hitchhiker's guide to diffusion tensor imaging. Frontiers in Neuroscience, 7(7 MAR), 1-14. https://doi.org/10.3389/fnins.2013.00031

Starrfelt, R., \& Shallice, T. (2014). What's in a name? The characterization of pure alexia. Cognitive Neuropsychology, 31(5-6), 367-77. https://doi.org/10.1080/02643294.2014.924226

Thiel, A., Herholz, K., Koyuncu, A., Ghaemi, M., Kracht, L. W., Habedank, B., \& Heiss, W.D. (2001). Plasticity of language networks in patients with brain tumors: A positron emission tomography activation study. Annals of Neurology, 50(5), 620-629. https://doi.org/10.1002/ana.1253

Thulborn, K. R., Carpenter, P. A., \& Just, M. A. (1999). Plasticity of language-related brain function during recovery from stroke. Stroke; a Journal of Cerebral Circulation, 30(4), 749-54.

Turkeltaub, P. E., Benson, J., Hamilton, R. H., Datta, A., Bikson, M., \& Coslett, H. B. (2012). Left lateralizing transcranial direct current stimulation improves reading efficiency. Brain Stimulation, 5(3), 201-7. https://doi.org/10.1016/j.brs.2011.04.002

Vigneau, M., Jobard, G., Mazoyer, B., \& Tzourio-Mazoyer, N. (2005). Word and non-word reading: what role for the Visual Word Form Area? NeuroImage, 27(3), 694-705. https://doi.org/10.1016/j.neuroimage.2005.04.038

Welcome, S. E., Pasquarella, A., Chen, X., Olson, D. R., \& Joanisse, M. F. (2014). Preserved mid-fusiform activation for visual words in a patient with a visual word recognition impairment. Neuropsychologia, 65, 113-24. https://doi.org/10.1016/j.neuropsychologia.2014.10.012

Woodhead, Z. V. J., Penny, W., Barnes, G. R., Crewes, H., Wise, R. J. S., Price, C. J., \& Leff, A. P. (2013). Reading therapy strengthens top-down connectivity in patients with pure alexia. Brain, 136(8), 2579-2591. https://doi.org/10.1093/brain/awt186

Yeatman, J. D., Rauschecker, A. M., \& Wandell, B. A. (2013). Anatomy of the visual word form area: adjacent cortical circuits and long-range white matter connections. Brain and Language, 125(2), 146-55. https://doi.org/10.1016/j.bandl.2012.04.010

\section{Figure Captions}

Fig 1 Schematic of block design of fMRI task 
Fig 2 Behavioral performance (reaction time) in the a) reading task and b) the word comparison task. Error bars indicate standard error

Fig 3 Patient A, extent of lesion shown in red on T1 image

Fig 4 Patient B, extent of lesion shown in red on T1 image

Fig 5 Statistical parametric maps of word (left) and letter-string (middle) and checkerboard (right) activation in the fMRI task in the healthy control volunteer. Color map indicated tvalue at height threshold $p<0.01$, in contrasts against implicit baseline

Fig 6 Probabilistic tractography seeded from the temporal occipital fusiform cortex in a.) Patient A, b.) Patient B and c.) the healthy control group. Left hemisphere tracts shown in blue, right hemisphere in orange

Fig 7 Probabilistic tractography seeded from the middle frontal gyrus in a.) Patient A, b.) Patient $B$ and c.) the healthy control group. Left hemisphere tracts shown in blue, right hemisphere in orange

Fig 8 Laterality index based on volume of tracts for regions of interest in Patient A, Patient B and 9 healthy control volunteers. Error bars indicated standard error. Negative values indicate greater volume in the right hemisphere. Temporal occipital fusiform cortex (TOFC); Lateral occipital cortex (LOC); Lingual gyrus (LG); Occipital pole (OP); Middle frontal gyrus (MFG) 


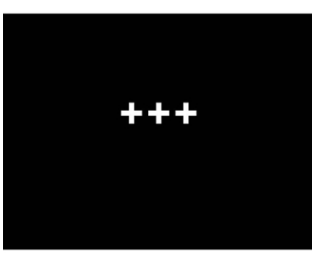

Fixation

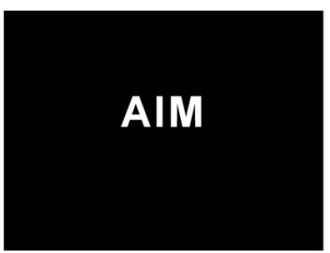

Words

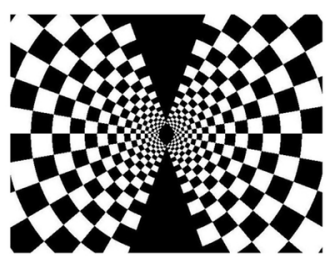

Checkerboard

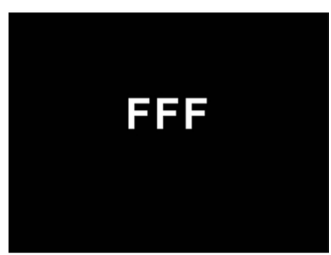

Letter Strings

1 Schematic of block design of fMRI task

$139 \times 56 \mathrm{~mm}(300 \times 300$ DPI $)$ 

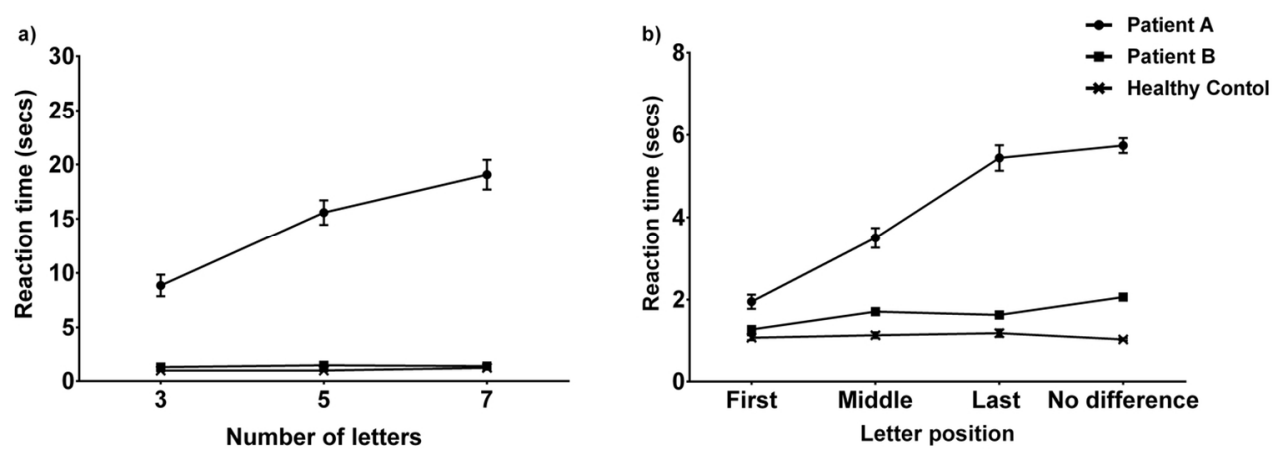

Behavioral performance (reaction time) in the a) reading task and b)the word comparison task. Error bars indicate standard error

$64 \times 23 \mathrm{~mm}(600 \times 600$ DPI $)$ 




Patient $A$, extent of lesion shown in red on T1 image $120 \times 21 \mathrm{~mm}(300 \times 300$ DPI $)$ 


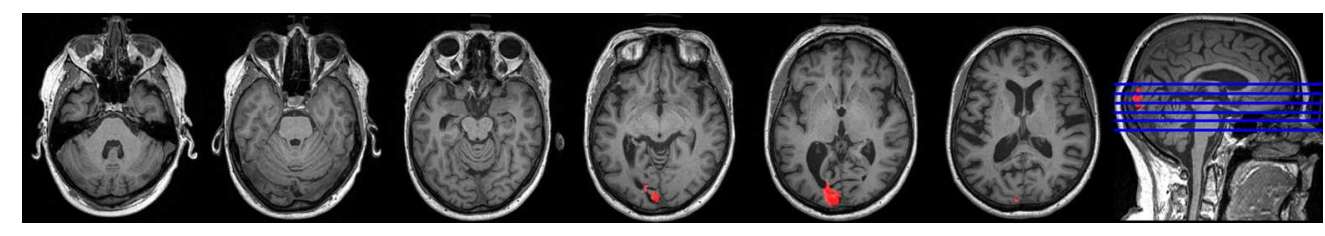

Patient $B$, extent of lesion shown in red on $T 1$ image

$117 \times 18 \mathrm{~mm}(300 \times 300 \mathrm{DPI})$ 

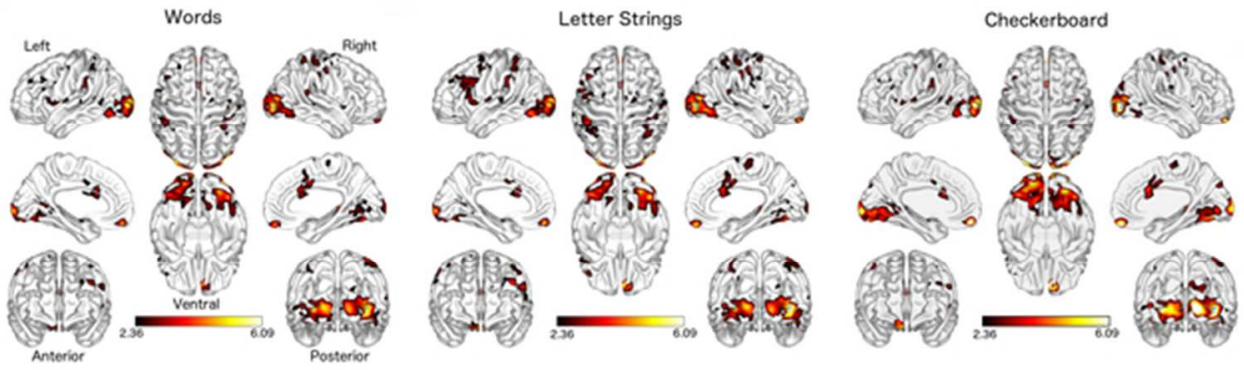

Statistical parametric maps of word (left) and letter-string (middle) and checkerboard (right) activation in the fMRI task in the healthy control volunteer. Color map indicated $t$-value at height threshold $p<0.01$, in contrasts against implicit baseline

$55 \times 17 \mathrm{~mm}(300 \times 300 \mathrm{DPI})$ 


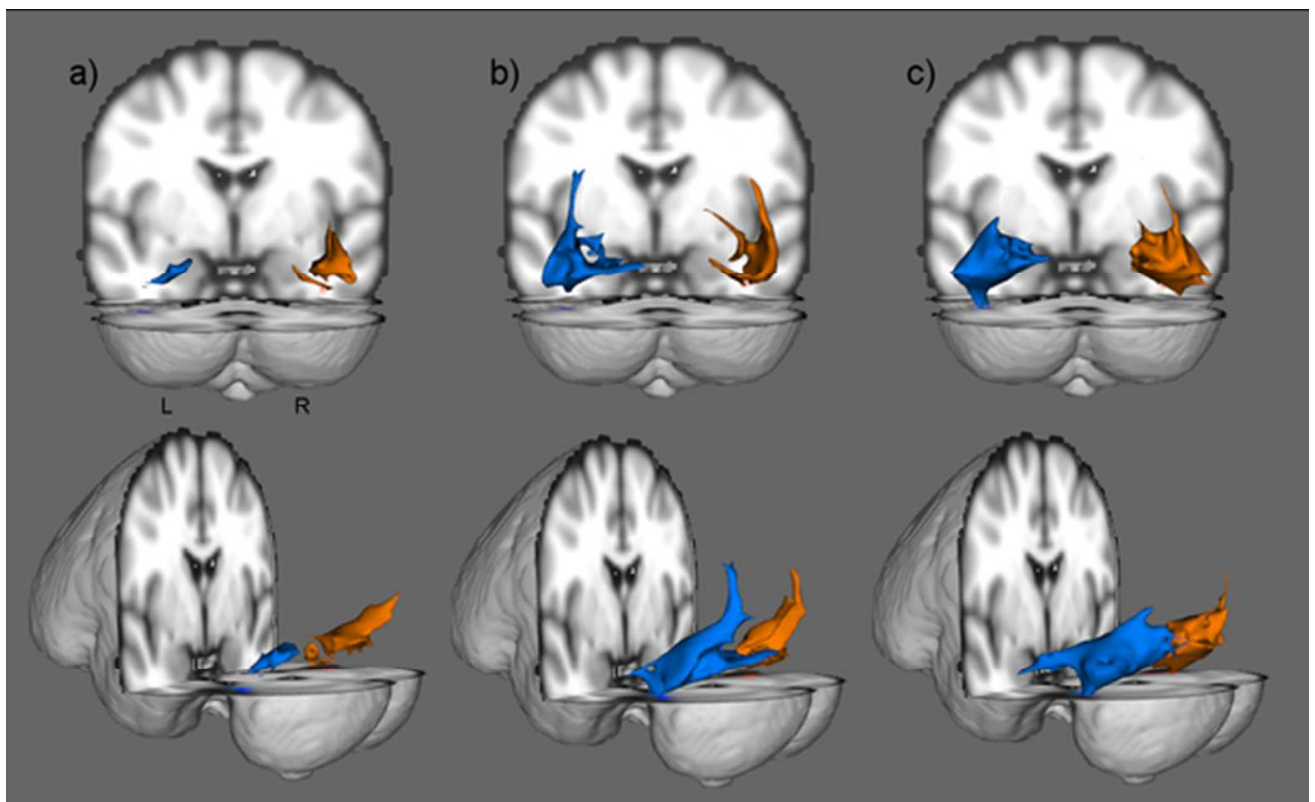

Probabilistic tractography seeded from the temporal occipital fusiform cortex in a.) Patient A, b.) Patient B and c.) the healthy control group. Left hemisphere tracts shown in blue, right hemisphere in orange $51 \times 31 \mathrm{~mm}(300 \times 300$ DPI $)$ 


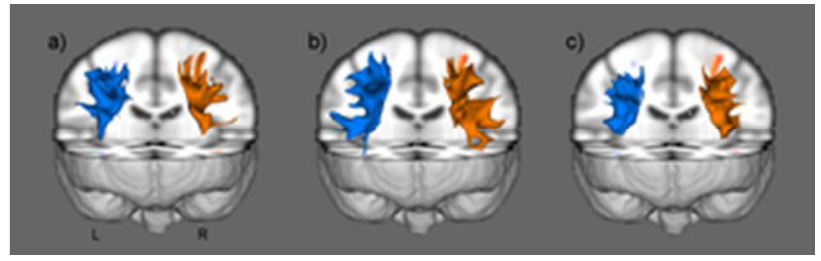

Probabilistic tractography seeded from the middle frontal gyrus in a.) Patient $A, b$.) Patient $B$ and c.) the healthy control group. Left hemisphere tracts shown in blue, right hemisphere in orange

$26 \times 8 \mathrm{~mm}(300 \times 300 \mathrm{DPI})$ 


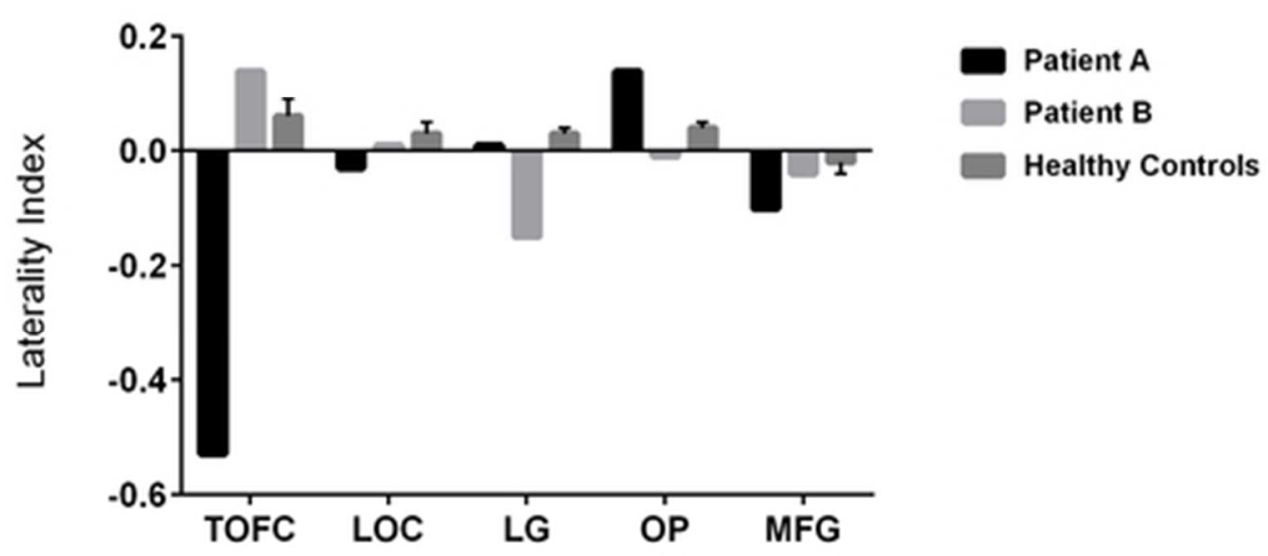

Seed region of interest

Laterality index based on volume of tracts for regions of interest in Patient A, Patient B and 9 healthy control volunteers. Error bars indicated standard error. Negative values indicate greater volume in the right hemisphere. Temporal occipital fusiform cortex (TOFC); Lateral occipital cortex (LOC); Lingual gyrus (LG); Occipital pole (OP); Middle frontal gyrus (MFG)

$21 \times 11 \mathrm{~mm}(600 \times 600 \mathrm{DPI})$ 